\title{
Risk-Constrained Bidding Strategy for a Joint Operation of Wind Power and Compressed Air Energy Storage Aggregators
}

\author{
Sahand Ghavidel, Student Member, IEEE, Mojtaba Jabbari Ghadi, Student Member, IEEE, Ali \\ Azizivahed, Student Member, IEEE, Jamshid Aghaei, Senior Member, IEEE, Li Li, Member, IEEE, \\ and Jiangfeng Zhang
}

\begin{abstract}
This paper proposes a coordinated strategy of a hybrid power plant (HPP) which includes a wind power aggregator (WPA) and a commercial compressed air energy storage (CAES) aggregator to participate in three electricity markets (day-ahead, intraday and balancing markets). The CAES aggregator has an extra ability which is called a simplecycle mode operation which makes it works like a gas turbine when is needed which helps the HPP to economically handle the miscalculations of the wind power and electricity price predictions. The coordinated strategy of the HPP is formulated as a three-stage stochastic optimization problem. To control the financial risks, the conditional value-at-risk model is added to the optimization problem. Moreover, the proposed offering method is capable of submitting both bidding quantity and curves to the day-ahead market. A mixed integer linear programming formulation is written for the problem which can be easily solved by commercially available software such as GAMS. The results which were tested on a realistic-based case study located in Spain show the applicability of the suggested method to increase the joint operation profit, and decrease the financial risks.
\end{abstract}

Index Terms - Hybrid power plant (HPP), Wind power aggregator (WPA), Compressed air energy storage (CAES), Mixed integer linear programming, Financial risks, Stochastic optimization problem.

\section{NOMENCLATURE}

\section{A. Definitions \\ CVaR Conditional Value-at-Risk \\ WPA Wind Power Aggregator \\ CAES Compressed Air Energy Storage \\ HPP Hybrid Power Plant}

S. Ghavidel, M. Jabbari Ghadi, A. Azizivahed, L. Li, J. Zhang are with Faculty of Engineering and Information Technology, University of Technology Sydney, PO Box 123, Broadway, NSW 2007, Australia (emails:\{Sahand.GhavidelJirsaraie, Mojtaba.JabbariGhadi, A.Azizivahed \}student.uts.edu.au, \{li.li, Jiangfeng.Zhang@uts.edu.au\}.

J. Aghaei is with the Department of Electrical and Electronics Engineering, Shiraz University of Technology, Shiraz, Iran (e-mails: aghaei@sutech.ac.ir).
B. Indices

$$
\begin{array}{cc}
\text { 1) } & \text { Sets } \\
s & \text { Index of scenario. } \\
t & \text { Index of time. } \\
w & \text { Index of wind power generation scenario. } \\
d & \text { Index of day-ahead market price scenario. } \\
i & \text { Index of intraday market price scenario. } \\
b & \text { Index of balancing market price scenario. } \\
2 \text { ) } & \text { Superscripts } \\
\text { Cha } & \text { Index for charge mode of CAES. } \\
D A & \text { Index for offered power to day-ahead market. } \\
\text { Dis } & \text { Index for discharge mode of CAES. } \\
\text { IN } & \text { Index for offered power to intraday market. } \\
\text { INT } & \text { Index for initial value. } \\
R e & \text { Index for real power for generating system. } \\
S C & \text { Index for scheduled power for generating system. } \\
\text { Sim } & \text { Index for simple-cycle mode of CAES. }
\end{array}
$$

C. Constants

$$
\begin{array}{cl}
N_{s} & \text { Total number of scenarios. } \\
N_{T} & \text { Total period of time. } \\
\pi_{s} & \text { Probability of occurrence of scenario } s . \\
\rho & \text { Price of energy in electricity market. } \\
\eta^{+/-} & \text {Positive/negative imbalance price ratio. } \\
\zeta & \text { Risk-aversion factor. } \\
\sigma & \text { Confidence level with } \sigma \in(0,1) . \\
P w^{\text {Max }} & \text { Maximum capacity of WPA. } \\
P c_{E x p / C o m}^{\text {Max }} & \text { Maximum expanding/compressing capacity of } \\
\Lambda & \text { CAES. } \\
E r & \text { CAES energy ratio for converting power to } \\
E c^{\text {Max/Min }} & \text { Maximum/minimum schedulable level of energy } \\
& \text { in CAES cavern. } \\
H c & \text { CEAS heat rate in one of operating modes. } \\
N G & \text { Natural gas price. }
\end{array}
$$

D. Variables
Ph Power produced by HPP.
$P w \quad$ Power produced by WPA.
$P C \quad$ Power produced by CAES. 
Positive/negative deviations of the generating $\varepsilon h^{+/-} \quad$ power of HPP from the scheduled power.

sh Total deviation of HPP power from the scheduled power.

$\varepsilon w^{+/-}$Positive/negative deviation of WPA power from the scheduled power.

$\varepsilon w$ Total deviation of WPA power from the scheduled power.

$O C_{t, s} \quad$ Total operational cost of CAES.

$\theta \quad$ Supplementary variable to calculate CVaR.

$\varphi_{s} \quad$ Continious non-negative variable to calculate CVaR.

Uc Binary variable to show ON/OFF operating status of CAES.

Ec Scheduled energy level of the CAES.

$V c^{\text {Exp/Com }}$ CAES variable operation and maintenance cost for expanding/compressing modes.

\section{INTRODUCTION}

$\mathrm{D}^{2}$ UE to the stochastic nature of the wind power input, it is generally very challenging for wind power aggregators (WPAs) to participate in electricity markets such as day-ahead and intra-day markets in a determined way and also to equally compete with non-renewable deterministic conventional power producers. Consequently, it is vital for WPAs to find new strategies to overcome these problems.

Currently, there is a significant amount of attention to energy storage systems mainly commercial compressed air energy storage (CAES) which is a developed technology with the ability to work as a gas turbine when is required [1]. Commercial CAES facilities can deliver an energy-shifting when there is a volatility in electricity market prices. Moreover, it is necessary to note that the capability of working as a gas turbine which is called simple-cycle mode operation, makes the CAES facilities to be different from other types of energy storages such as batteries or normal CAES itself. CAES with simple-cycle mode operation can follow the everyday scheduling in a more enhanced way and exploit price spikes when is more desirable [2].

\section{A. Literature Review and Approach}

Recently, numerous studies focus on self-scheduling approaches for CAES facilities and aggregators and analyze the energy trading in different types of electricity markets to maximize their profit [3]. For instance, a co-optimized CAES model to clarify the importance of providing operating reserves and energy arbitrage in diverse electricity markets located in the united states is presented in [4]. Ref. [2] offers a risk-constrained bidding strategy for a commercial CAES aggregator that participates in a day-ahead (DA) market. The CAES used in this study has an additional facility called simple-cycle mode which works as an extra gas turbine.

On the other hand, many studies concentrate on the best offering strategies for WPAs to contribute in different types of electricity markets [5]. For example, in [6], a method is suggested to advance an offering strategy for a WPA participating in different electricity markets considering the uncertainties of the wind power and electricity market prices. Ref. [7] offers a strategy for a WPA to participate in a DA market by considering the WPA as a price-maker producer. Ref. [8] also offers a strategy for a WPA as a price-taker producer in a DA market, but as a price-maker producer in the real time (balancing) market. Ref. [9] presents a new model considering the uncertainties of the wind power and loads for corrective voltage control to handle the condition when power systems have voltage instability because of an unexpected failure and contingencies.

Besides, a variety of studies offer strategies for WPAs in conjunction with other types of aggregators [10-12]. For example, Ref. [13] analyses the combined operation of a pumped-storage unit and a WPA. The uncertainties included in this study are the wind power and market prices fluctuations. In this regard, a very similar study is done in [4] which evaluates the impact of wind power uncertainties in a joint operation with a pumped-hydro aggregator. Ref. [14] offers a bidding strategy for a hydro aggregator and a WPA to contribute in a DA market. In this study, in order to control the financial risk, the conditional value-at-risk (CVaR) is added to the model. Ref. [15] assesses two separate models comprising of joint operation of a WPA and a gas turbine aggregator, as well as a WPA and a CAES aggregator. An offering strategy for a WPA along with a flexible load (demand response provider) is provided in [16] which helps the WPA to handle the wind uncertainties. With the intention of minimizing the operational cost and imbalance payments due to the wind power imbalances, an offering strategy for controlling critical peak pricing events is assessed in [17]. The strategy is done from the viewpoint of a demand response aggregator which owns a wind facility. Ref. [18] provides a bidding strategy for a WPA and a demand response provider to contribute in intraday market along with DA and balancing markets.

\section{B. Contribution}

This paper proposes a framework in which a WPA can compensate its deviation between the actual and forecasted value of wind generation by the coordinated operation with a commercial CAES aggregator in the form of an HPP. As can be seen from Fig. 1, HPP gathers information from the WPA and CAES, and takes part in DA, intraday and balancing markets. The uncertainties of the WPA production and the three mentioned market prices are stochastically considered using the stochastic programming method. To find the best bidding strategy which also controls the financial risk, the $\mathrm{CVaR}$ is added to the stochastic programming model. Note that this paper is an extended version of a conference paper presented on Australasian Universities Power Engineering Conference (AUPEC), 2017 [19].

The contributions of the paper are given as follows:

- To model a CAES which is equipped with a simple-cycle mode of operation and thus able to work like a gas turbine.

- To develop a risk-constrained optimal offering strategy 
model for a HPP consisting of a CAES aggregator and a WPA so as to maximize their expected profit and also to mitigate wind power uncertainties in DA, intraday and balancing markets.

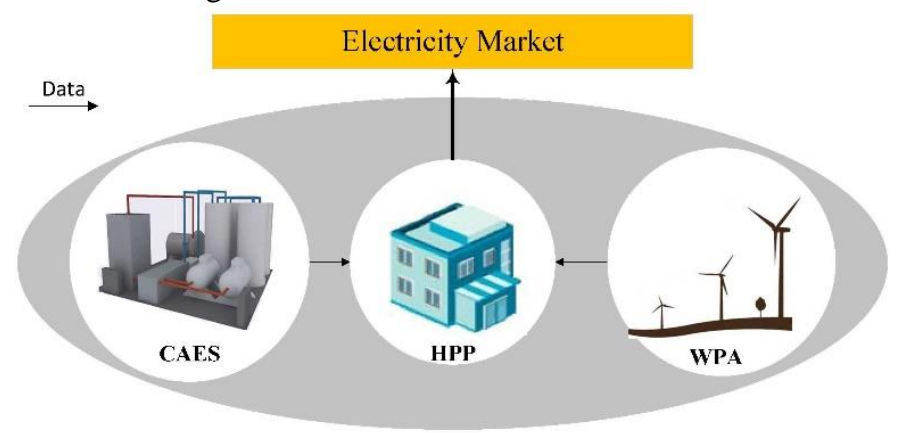

Fig. 1. Diagram illustration of three configurations

\section{Paper Organization}

The rest of the paper is organized as follows: Section II provides the problem formulation of three configurations including CAES aggregator, WPA, and HPP. Section IV discusses the case study and provides the results. Finally, Section V concludes the paper.

\section{PROBLEM Formulation}

\section{A. CAES Aggregator Modeling}

In this section, a bidding strategy for a CAES aggregator to participate in the electricity markets is modeled as a stochastic optimization problem, in which the goal is to maximize the profit which includes the total profits of the CAES aggregator from the electricity markets minus its operational costs. From the reliability point of view, CAES has very highly reliable performance and output in comparison with other producers like WPA [2]. Therefore, there is no need for CAES aggregator to participate in the balancing market. In this case, two electricity markets including DA and intraday are considered in this paper for the modeling of CAES aggregator. About the two mentioned electricity markets, the CAES operational cost and considering the $\mathrm{CVaR}$ to control the financial risks, the objective function of the optimization problem can be written as follows:

$$
\begin{aligned}
\operatorname{Max}_{\Theta c_{t, s} ; \varphi_{s}, \forall s ; \theta}\left[Z_{P r o f i t}^{C A E S}\right] & \\
= & \sum_{s=1}^{N_{S}} \sum_{t=1}^{N_{T}} \pi_{s}\left[\rho_{t, s}^{D A} \cdot P c_{t, s}^{D A}+\rho_{t, s}^{I N} \cdot P c_{t, s}^{I N}\right. \\
& \left.-O C_{t, s}\right]+\zeta\left(\theta-\frac{1}{(1-\sigma)} \sum_{s=1}^{N_{s}} \pi_{s} \varphi_{s}\right)
\end{aligned}
$$

where $\Theta c_{t, s}=\left\{P c_{t, s}^{D A}, P c_{t, s}^{I N}, P c_{t, s}^{S C}, P c_{t, s}^{S C, D i s}, P c_{t, s}^{S C, S i m}, P c_{t, s}^{S C, C h a}\right.$ $\left., U c_{t, s}^{S C, D i s}, U c_{t, s}^{S C, S i m}, U c_{t, s}^{S C, C h a}, E c_{t, s}^{S C}, \forall t, \forall s\right\}$ are the variables of the CAES aggregator optimization problem. The objective function has two general terms including the expected profit of CAES aggregator, which is the difference between its revenue and operating cost, and the CVaR (i.e., multiplied by the risk-

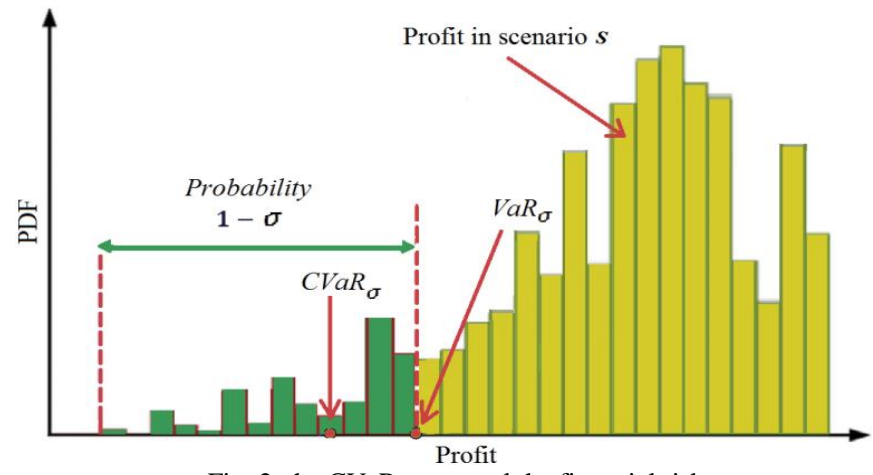

Fig. 2. the $\mathrm{CVaR}$ to control the financial risks

aversion factor $\zeta$ ). The revenue/cost of CAES aggregator is obtained from selling energy in DA and intraday markets; while its costs is sum of the purchased energy to charge the air cavern plus variable costs of simple-cycle mode. The terms $\rho_{t, s}^{D A} \cdot P c_{t, s}^{D A}$ and $\rho_{t, s}^{I N} \cdot P c_{t, s}^{I N}$ express the revenue/cost from the selling/purchasing of energy in CAES in DA and intraday markets, respectively. The term $O C_{t, s}$ in the objective function is related to the CAES operational cost which is computed according to the different CAES operating modes (charging, discharging and simple-cycle modes) and their amount of power. The last term is for modeling of the $\mathrm{CVaR}$, and it is multiplied by $\zeta$ which has the values between 0 and 1 , and the value 0 represents financial risk is not considered while 1 fully considers the financial risk. The $\mathrm{CVaR}$ signifies the expected profit of the $(1-\sigma) \times 100$ percent of scenarios yielding the lowest profits, and it is used to regulate the risk due to profit variability confronted by the CAES aggregator. Noteworthy, as shown in Fig. 2, $(1-\sigma)$ regulates the area of the profit distribution function covering the least profitable scenarios. Note that $\theta$ is an auxiliary variable, and its value is simultaneously optimized along with variables $\Theta c_{t, s}$ and $\varphi_{s}$, where $\varphi_{s}$ is a continuous non-negative variable equal to the maximum of $\theta-\sum_{t=1}^{N_{T}}\left[\rho_{t, s}^{D A} \cdot P c_{t, s}^{D A}+\rho_{t, s}^{I N} P c_{t, s}^{I N}-O C_{t, s}\right]$ and 0 . More information about CVaR modeling is provided in [20, 21].

The objective function defined in (1) is subject to the following constraints [2]:

$$
\begin{gathered}
-P c_{C o m}^{M a x} \leq P c_{t, s}^{\beta} \leq P c_{E x p}^{M a x} \quad \forall t, s, \quad \beta=D A, S C \\
-\Lambda . P c_{C o m}^{M a x} \leq P c_{t, s}^{I N} \leq \Lambda \cdot P c_{E x p}^{M a x} \quad \forall t, \forall s \\
P c_{t, s}^{S C}=P c_{t, s}^{D A}+P c_{t, s}^{I N} \quad \forall t, \forall s \\
P c_{t, s}^{S C}=P c_{t, s}^{S C, D i s}+P c_{t, s}^{S C, S i m}-P c_{t, s}^{S C, C h a} \quad \forall t, s \\
0 \leq P c_{t, s}^{S C, \varpi} \leq P c_{E x p}^{M a x} \cdot \mathrm{U} c_{\mathrm{t}, s}^{S C, \varpi} \forall \mathrm{t}, \mathrm{s}, \quad \varpi=D i s, \text { Sim, Cha } \\
U c_{t, s}^{S C, D i s}+U c_{t, s}^{S C, S i m}+U c_{t, s}^{S C, C h a} \leq 1 \quad \forall t, s \\
E c_{t, s}^{S C}=E c_{t-1, s}^{S C}+E r\left(P c_{t, s}^{S C, C h a}-P c_{t, s}^{S C, D i s}\right) \quad \forall t \\
>1, \forall s \\
E c_{t, s}^{S C}=E c^{I N T} \quad t=1, \forall s \\
E c^{M i n} \leq E c_{t, s}^{S C} \leq E c^{M a x} \quad \forall t, s
\end{gathered}
$$




$$
\begin{aligned}
& O C_{t, s}=P c_{t, s}^{S C, D i s}(\left.H c^{D i s} \cdot N G+V c^{E x p}\right) \\
&+P c_{t, s}^{S C, S i m}\left(H c^{S i m} \cdot N G+V c^{E x p}\right. \\
&\left.+V c^{C o m}\right) \\
&+P c_{t, s}^{S C, C h a} V c^{C o m} \quad \forall t, s \\
&-\sum_{t=1}^{N_{T}}\left[\rho_{t, s}^{D A} \cdot P c_{t, s}^{D A}\right.\left.+\rho_{t, s}^{I N} P c_{t, s}^{I N}-O C_{t, s}\right]+\theta-\varphi_{s} \\
& \leq 0 \quad \forall s \\
& \varphi_{s} \geq 0 \quad \forall s \\
&\left(P c_{t, s}^{D A}-P_{t, s^{\prime}}^{D A}\right) \cdot\left(\rho_{t, s}^{D A}-\rho_{t, s^{\prime}}^{D A}\right) \geq 0 \quad \forall t, s, s^{\prime} \\
& P c_{t, s}^{D A}=P c_{t, s^{\prime}}^{D A} \quad \forall t, s, s^{\prime} \quad: \rho_{t, s}^{D A}=\rho_{t, s^{\prime}}^{D A}
\end{aligned}
$$

where (2) limits the offering CAES power to the DA market and its scheduled power, respectively. Note that both of $P c_{t, s}^{D A}$ and $P c_{t, s}^{S C}$ in (2) can in each hour has positive or negative power values which means that the CAES aggregator has the capability of either buying or selling energy in each hours of the day. The amount of CAES aggregator power to participate in the intraday market is also limited to $\Lambda$ multiply by its participation capacity to the DA market as formulated in (3). In other words, according to (3), CAES is not allowed to use its full capacity to participate in the intraday market even it is more economical [20]. The total scheduled CAES power which is based on the summation of CAES bidding powers to DA and intraday electricity markets is expressed in (4). Equation (5) shows that the CAES scheduled power is restricted by three mentioned CAES working modes (i.e. discharging, simple-cycle or charging modes). The restrictions of CAES power in these modes are formulated in (6). It is worthwhile to mention that at each period of time and scenario, the CAES can only work in one of the charging, discharging, or simple-cycle modes. This limitation can be precisely specified in (7). The scheduled CAES energy level which is also called the state-transition equation is expressed in (8) and also the initial value of this energy is limited by (9). This energy is also restricted by the capacity of CAES cavern as mathematically formulated in (10). Equation (11) formulates the operational cost of CAES which is used in the objective function (1). The CAES operational cost is computed according to the different CAES operating modes (charging, discharging and simple-cycle modes) and their amount of power. In order to calculate and control the functional risk, (12) and (13) are required for the model.

The stochastic programming model (1)-(13) can be solved to attain the optimal quantities to be submitted in the dayahead market. However, it is more appropriate to develop optimal offering curves for every hour of this market. For this purpose, variable $P c_{t}^{D A}$, which are the power traded in the dayahead market for each time period $t$, is considered to be dependent on scenarios $\left(P c_{t}^{D A} \rightarrow P c_{t, s}^{D A}\right)$ and the constraints (14) and (15) are added to model (1)-(13). Constraints (14) make offering curves non-decreasing which is an obligation in most electricity markets. Equations (15) are non-anticipativity constraints, which enforce the idea that only one offering curve can be submitted to the day-ahead market regardless of the imbalance price and actual wind power generation. Note that the bidding strategy model remains decomposable at each time period even constraints (14) and (15) are included.

\section{B. WPA Modeling}

In this section, the bidding strategy of the WPA is formulated for profit maximization. Three electricity markets including DA, intraday and balancing markets are considered in this model, as shown in Fig. 3 [22]. Note that, as it can be seen in Fig. 3, the intraday market remains two and a half hours before the balancing market. With regard to the three

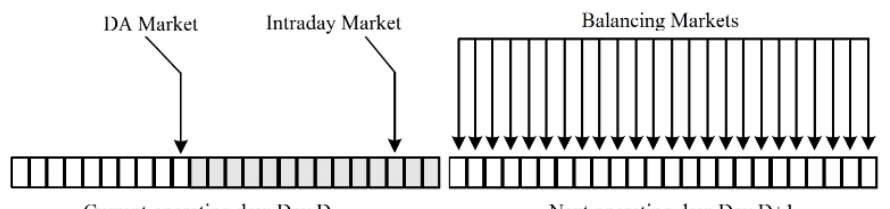

Current operating day: Day D Next operating day: Day D+1

Fig. 3. Three electricity markets framework

mentioned electricity markets and considering the $\mathrm{CVaR}$ to control the financial risks, the objective function of the optimization problem can be written as (16) [22].

$$
\begin{aligned}
\operatorname{Max}_{\Theta w_{t, s} ; \varphi_{s}, \forall s ; \theta}[ & \left.Z_{\text {Profit }}^{\text {WPA }}\right] \\
& =\sum_{s=1}^{N_{s}} \sum_{t=1}^{N_{T}} \pi_{s}\left[\rho_{t, s}^{D A} \cdot P w_{t, s}^{D A}+\rho_{t, s}^{I N} \cdot P w_{t, s}^{I N}\right. \\
& \left.+\rho_{t, s}^{D A} \cdot \eta_{t, s}^{+} \cdot \varepsilon w_{t, s}^{+}-\rho_{t, s}^{D A} \cdot \eta_{t, s}^{-} \cdot \varepsilon w_{t, s}^{-}\right] \\
& +\zeta\left(\theta-\frac{1}{(1-\sigma)} \sum_{s=1}^{N_{s}} \pi_{s} \varphi_{s}\right)
\end{aligned}
$$

where $\Theta w_{t, s}=\left\{P w_{t, s}^{D A}, P w_{t, s}^{I N}, P w_{t, s}^{S C}, \varepsilon w_{t, s}^{+}, \varepsilon w_{t, s}^{-}, \quad \forall t, s\right\}$ are the variables related to the WPA optimization problem. The objective function is composed of the expected profit of WPA, and the CVaR which is multiplied by the risk-aversion factor $\zeta$. The total revenue/cost of WPA comes from the following: firstly, selling energy in the DA market; secondly, selling/purchasing energy in the intraday market and thirdly, the revenue/cost of participation in the balancing market due to positive/negative scheduling deviations from the actual generated power of producer. In (16), the terms $\rho_{t, s}^{D A} \cdot P w_{t, s}^{D A}$ and $\rho_{t, s}^{I N} . P w_{t, s}^{I N}$ state the revenue from DA market and revenue/cost from intraday market, respectively; while the terms $\rho_{t, s}^{D A} \cdot \eta_{t, s}^{+} \cdot \varepsilon w_{t, s}^{+}$and $\rho_{t, s}^{D A} \cdot \eta_{t, s}^{-} \cdot \varepsilon w_{t, s}^{-}$indicate the revenue/cost from the positive/negative energy deviations in the balancing market. The last term in (16) is related to considering the CVaR to control the financial risks.

The objective function of profit maximization of WPA in (16) is subject to some important constraints as follows [22]:

$$
\begin{gathered}
0 \leq P w_{t, s}^{\beta} \leq P w^{\operatorname{Max}} \quad \forall t, s, \quad \beta=D A, S C \\
0 \leq P w_{t, s}^{I N} \leq \Lambda . P w_{t, s}^{D A} \quad \forall t, s \\
P w_{t, s}^{S C}=P w_{t, s}^{D A}+P w_{t, s}^{I N} \quad \forall t, s
\end{gathered}
$$

where (17) limits the offering power of WPA to the DA market and its scheduled power. Note that $P w_{t, s}^{D A}$ and $P w_{t, s}^{S C}$ in (17) can only be positive values. In other words, WPA can 
only sell electricity to the DA market and have positive value for its scheduled power. The amount of WPA power to participate in the intraday market is also limited just like CAES aggregator to $\Lambda$ multiply by its participation capacity to the DA market as formulated in (18). The total scheduled powers of WPA is also limited to its participation in DA and intraday offers as formulated in (19) [22].

$$
\begin{gathered}
\varepsilon w_{\mathrm{t}, s}=P w_{t, s}^{R e}-P w_{t, s}^{S C} \quad \forall t, \forall s \\
\varepsilon w_{t, s}=\varepsilon w_{t, s}^{+}-\varepsilon w_{t, s}^{-} \quad \forall t, \forall s \\
0 \leq \varepsilon w_{t, s}^{+} \leq P w_{t, s}^{R e} \quad \forall t, \forall s \\
0 \leq \varepsilon w_{t, s}^{-} \leq P w^{\text {max }} \quad \forall t, \forall s \\
-\sum_{t=1}^{N_{T}}\left[\rho_{t, s}^{D A} \cdot P w_{t, s}^{D A}+\rho_{t, s}^{I N} \cdot P w_{t, s}^{I N}+\rho_{t, s}^{D A} \cdot \eta_{t, s^{\prime}}^{+} \cdot \varepsilon w_{t, s}^{+}\right. \\
\left.-\rho_{t, s}^{D A} \cdot \eta_{t, s^{\prime}}^{-} \cdot \varepsilon w_{t, s}^{-}\right]+\theta-\varphi_{s} \\
\leq 0 \quad \forall s \\
\varphi_{s} \geq 0 \quad \forall s \\
\left(P w_{t, s}^{D A}-P w_{t, s^{\prime}}^{D A}\right) \cdot\left(\rho_{t, s}^{D A}-\rho_{t, s^{\prime}}^{D A}\right) \geq 0 \quad \forall t, s, s^{\prime} \\
P w_{t, s}^{D A}=P w_{t, s^{\prime}}^{D A} \quad \forall t, s, s^{\prime}: \rho_{t, s}^{D A}=\rho_{t, s^{\prime}}^{D A}
\end{gathered}
$$

The total negative and positive imbalances according to the amount of scheduled and actual wind power productions are formulated in (20) to (23). Constraints (24) and (25) formulates the required limitations for the CVaR calculation. (26) and (27) are used with the aim of offering non-decreasing curves to the DA electricity market.

\section{HPP Modeling}

In this section, a bidding strategy for the HPP is modeled to achieve the maximum profit of joint operation of CAES aggregator and WPA. All three electricity markets used in WPA modeling as shown in Fig. 3 are also taken into consideration in HPP modeling [22]. Taking into consideration the $\mathrm{CVaR}$ model, the objective function for the participation of HPP in all given electricity markets can be written as follows:

$$
\begin{aligned}
\operatorname{Max}_{\Theta h_{t, s} ; \varphi_{s}, \forall s ; \theta}\left[Z_{\text {Profit }}^{H p p}\right] & \\
& =\sum_{\substack{s=1 \\
N_{S}}}^{N_{T}} \sum_{t=1} \pi_{s}\left[\rho_{t, s}^{D A} \cdot P h_{t, s}^{D A}+\rho_{t, s}^{I N} \cdot P h_{t, s}^{I N}\right. \\
& +\rho_{t, s}^{D A} \cdot \eta_{t, s}^{+} \cdot \varepsilon h_{t, s}^{+}-\rho_{t, s}^{D A} \cdot \eta_{t, s}^{-} \cdot \varepsilon h_{t, s}^{+} \\
& \left.-O C_{t, s}\right]+\zeta\left(\theta-\frac{1}{(1-\sigma)} \sum_{s=1}^{N_{s}} \pi_{s} \varphi_{s}\right)
\end{aligned}
$$

where $\Theta h_{t, s}=\left\{P h_{t, s}^{D A}, P h_{t, s}^{I N}, P h_{t, s}^{S C}, \varepsilon h_{t, s}^{+}, \varepsilon h_{t, s}^{+}, P w_{t, s}^{D A}, P c_{t, s}^{D A}, P w_{t \omega}^{I N}\right.$, $P c_{t, s}^{I N}, P w_{t, s}^{S C}, P c_{t, s}^{S C} P c_{t, s}^{S C, D i s}, P c_{t, s}^{S C, S i m}, P c_{t, s}^{S C, C h a}, U c_{t, s}^{S C, D i s}, U c_{t, s}^{S C, S i m}$, $\left.U c_{t, s}^{S C, C h a}, E c_{t, s}^{S C} \quad \forall t, s\right\}$ are the variables related to the HPP optimization problem. As it can be seen from (28), two general expressions of the objective function are included the expected profit of HPP (i.e. as the result of market transactions and operational cost) and the CVaR. The revenue/cost of HPP comes from selling/purchasing energy in both of the DA and intraday markets as well as the revenue/cost from the positive/negative energy deviations in the balancing market. In (28), the terms $\rho_{t, s}^{D A} \cdot P h_{t, s}^{D A}$ and $\rho_{t, s}^{I N} \cdot P h_{t, s}^{I N}$ refer to the revenue/cost of HPP from the DA and intraday markets, and the terms $\rho_{t, s}^{D A} \cdot \eta_{t, s}^{+} \cdot \varepsilon h_{t, s}^{+}$and $\rho_{t, s}^{D A} \cdot \eta_{t, s}^{-} \cdot \varepsilon h_{t, s}^{-}$indicate the revenue/cost from the positive/negative energy deviations in the balancing market. The term $O C_{t, s}$ in the objective function is related to the CAES operational cost which is computed according to the different CAES operating modes (charging, discharging and simple-cycle modes) and their amount of power; see (11). The last term in the HPP modeling objective function is for modeling of the $\mathrm{CVaR}$ which is multiplied by $\zeta$ with the values between 0 for not considering the financial risk and 1 for fully considering the financial risk.

The objective function of HPP optimization problem (28) is subject to some joint constraints associated with both of the WPA and CAES providers, some constraints associated specifically with for modeling of CAES aggregator, and some only for the WPA model.

The constraints of HPP associated with the CAES model are defined previously in the CAES modeling section; see (2) to (11). The constraints of HPP associated specifically with the WPA model are also previously defined in in the WPA modeling section; see (17) to (23).

The joint constraints associated with both of both of the WPA and CAES providers to function as an HPP are defined as bellow:

$$
\begin{gathered}
P h_{t, s}^{\beta}=P w_{t, s}^{\beta}+P c_{t, s}^{\beta} \quad \forall t, s, \quad \beta=D A, S C, I N \\
P h_{t, s}^{S C}=P h_{t, s}^{D A}+P h_{t, s}^{I N} \quad \forall t, s \\
\varepsilon h_{t, s}=P w_{t, s}^{R e}+P c_{t, s}^{R e}-P h_{t, s}^{S C} \quad \forall t, s \\
\varepsilon h_{t, s}=\varepsilon h_{t, s}^{+}-\varepsilon h_{t, s}^{-} \quad \forall t, s \\
0 \leq \varepsilon h_{t, s}^{+} \leq P w_{t, s}^{R e}+P c_{t, s}^{R e} \quad \forall t, s \\
0 \leq \varepsilon h_{t, s}^{-} \leq P w^{M a x}+P c_{E x p}^{M a x} \quad \forall t, s \\
-\sum_{t=1}^{N_{T}}\left[\rho_{t, s}^{D A} \cdot P h_{t, s}^{D A}+\rho_{t, s}^{I N} P h_{t, s}^{I N}+\rho_{t, s}^{D A} \cdot \eta_{t, s^{\cdot}}^{+} \varepsilon h_{t, s}^{+}\right. \\
\left.-\rho_{t, s}^{D A} \cdot \eta_{t, s}^{-} \cdot \varepsilon h_{t, s}^{-}\right]+\theta-\varphi_{s} \leq 0 \quad \forall s \\
\varphi_{s} \geq 0 \quad \forall s \quad \forall \quad \rho_{t, s} \\
\left(P h_{t, s}^{D A}-P h_{t, s^{\prime}}^{D A}\right) \cdot\left(\rho_{t, s}^{D A}-\rho_{t, s^{\prime}}^{D A}\right) \geq 0 \quad \forall t, s^{\prime} \\
P h_{t, s}^{D A}=P h_{t, s^{\prime}}^{D A} \quad \forall t, s, s^{\prime}: \rho_{t, s}^{D A}=\rho_{t, s^{\prime}}^{D A}
\end{gathered}
$$

where (29) limits DA and intraday offers and the total scheduled power of HPP. The total scheduled power of HPP should be equal to its DA and intraday offers which is mathematically formulated in (30). The total negative and positive imbalances according to the HPP scheduled power and the actual wind and CAES powers in the time of balancing market are formulated in (31) to (34). As previously mentioned, unlike the WPA, the CAES aggregator is very reliable and has a deterministic output. For that reason, in this paper, it is supposed that the actual CAES powers in the time of balancing market are equal to its power that has been 
scheduled. Similar to WPA and CAES modeling, constraints (35) and (36) are formulated for CVaR calculation, and (37) and (38) are defined for obtaining the non-decreasing DA market offering curves.

\section{WIND GENERATION AND MARKET PRICES MODELING}

The wind power and market price uncertainties are modeled as follows: $N_{1}, N_{2}, N_{3}$ and $N_{4}$ scenarios are generated for wind power generation, day-ahead market, intraday market and balancing market prices, respectively. These uncertainty sources are separated into two categories; 1) wind power generation $(P w(w))$ and day-ahead market price $\left(\rho^{D A}(d)\right)$ scenarios which are independent uncertainty parameters (hereand-now), 2) the intraday market price $\left(\rho^{I N}(d, i)\right)$ scenarios which are feasible for each possible realization of day-ahead market price scenarios. In other words, the intraday market price scenarios are generated based on day-ahead market price scenarios. Similarly, the balancing market price $\left(\eta^{+}(b)\right.$, $\left.\eta^{-}(b)\right)$ scenarios are generated based on each possible wind

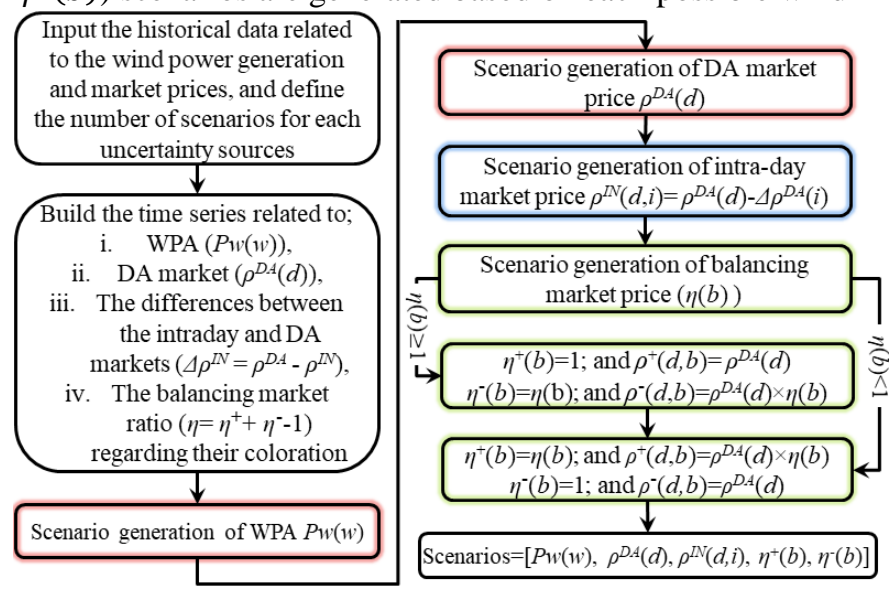

Fig. 4. Flowchart of stochastic variable modeling process

power generation and day-ahead market price scenarios.

Due to a dependency of intraday and balancing market prices on wind power generation and day-ahead market price, the correlation among these stochastic variables are defined as $\left(\rho^{D A}-\rho^{I N}\right)$ and $\left(\eta^{+}+\eta^{-}-1\right)$ for all scenarios. Also, the symmetric scenario tree is implemented to construct the $N_{S}=$ $N_{1} \times N_{2} \times N_{3} \times N_{4}$ scenarios based on the independent and dependent scenarios.

Fig. 4 shows the flowchart of the stochastic modeling process which leads to the generation of all scenarios $\left(P w(w), \rho^{D A}(d),\left(\rho^{I N}(d, i),\left(\eta^{+}(b), \eta^{-}(b)\right)\right.\right.$. It is worthy to mention that the red highlighted section indicates the first category uncertainty source (i.e., the wind power generation and day-ahead market price scenarios), and the blue and green highlighted section indicate the second category uncertainty source (i.e., the intraday and balancing market price scenarios). After scenario generation of balancing market prices $\eta(b)$, the highlighted green color part of Fig. 4 shows that there are two ways of calculating the balancing market prices $\left(\eta^{+}(b), \eta^{-}(b)\right)$ based on the values of $\eta(b)$.

\section{CASE Study AND Results}

The intention of the offered method is to manage and bring together a WPA and CAES producer to be working as an HPP to contribute in three electricity markets. The uncertainties of wind power and market prices are produced with a set of scenarios using an adapted hybrid neural network and a hybrid Jaya algorithm [23].

The offered methodology is applied to the Sotavento (Spain) experimental wind farm, with the maximum capacity of $26.54 \mathrm{MW}$ [24]. In order to train the artificial neural network, the wind power historical data of the year 2010 are used. The scenarios related to market prices are derived by a three-step process: First, market prices are predicted for 30 days using an adapted hybrid neural network and a hybrid Jaya algorithm [22, 23], and the error probability distribution function (PDF) is estimated for each hours (i.e., 24 PDFs in this study). Next, according to the estimated PDFs, immense numbers of scenarios are generated by implementing the roulette wheel mechanism. Finally, the scenario reduction technique (the fast forward algorithm) is implemented in order to reduce the number of scenarios by eliminating the similar scenarios and very low probable scenarios [25].

The historical data of market prices are also derived based on the Iberian Peninsula electricity market [26]. The uncertainties of the problem are modeled through a scenario tree with 3000 scenarios $(10 \times 5 \times 6 \times 10)$ including ten, five, six and, ten scenarios for DA, intraday, balancing market prices and the wind generation, respectively. The simulation results are presented for the 12th of March, 2010. Note that if there is not much historical data available, some other types of methods such as robust optimization or information gap theory can be used to solve the optimization problem [27, 28].

The CAES heat rate for the discharging and simple-cycle mode is considered to be 0.4185 and 0.837 , respectively in which the simple-cycle mode heat rate has double value. The cost of expander and compressor operation and maintenance are similarly selected to be $0.87 € / \mathrm{kWh}$. The air storage level of cavern is limited between 1 and $15 \mathrm{MW}$. The initial air storage level of cavern and the energy ratio of CAES are equal to $1 \mathrm{MW}$ and 0.95 , respectively. Also, the natural gas price is equal to be $3.5 € / G J$.

The upper bound level of CAES and wind power production for the intraday market are similarly considered to be equal to 30 percent of their DA market production level. Also, in the CVaR calculation, the confidence level $\sigma$ is given to be equal to 0.95 .

The offered methodology is initially applied to the MATLAB software to generate the wind power and market price scenarios. After reducing the number of scenarios by the scenario reduction technique, the scenario data are used as an input to the GAMS software. Note that the CPLEX solver is used in the GAMS software to solve the optimization problem. All the simulations of the study are performed in less than 120.756 seconds on a $2.3 \mathrm{GHz}$ Intel ${ }^{\circledR} \mathrm{CORE}^{\mathrm{TM}} \mathrm{i} 5$ laptop with 8 GB of RAM.

In this paper, five cases are considered to evaluate the 
applicability and effectiveness of the proposed approach as follows:

\section{A. Case I: Base Case}

In the base case, the WPA and CAES producers are coordinated to participate in three electricity markets. In this case, the CAES simple-cycle mode operation is not considered by not adding it to the formulation. The offering day-ahead market curves are also not considered by not including (14), (26) and (37) in the optimization problems of WPA, CAES and HPP modeling. Moreover, the CVaR is not considered in the base case by giving 0 value to the risk factor $\zeta$.

Fig. 5 depicts the comparison of optimal energy hourly bids in the DA market for three different configurations including WPA only, CAES aggregator only, and HPP. It can be seen

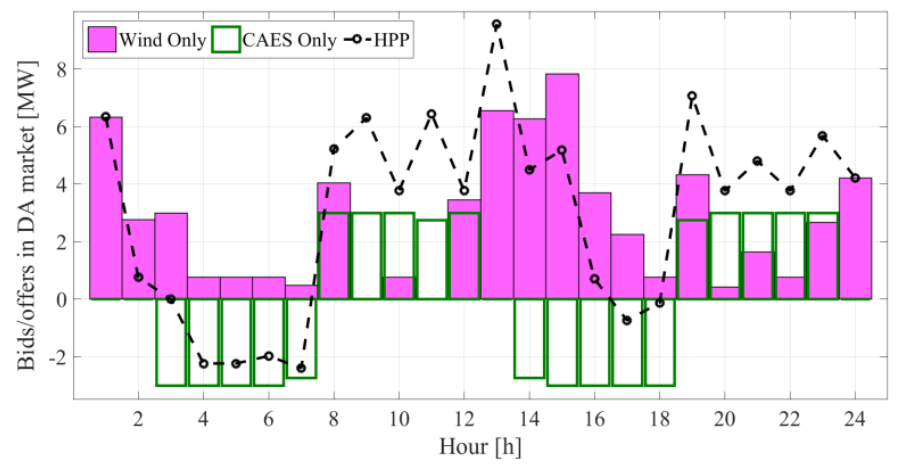

Fig. 5. Optimal hourly power bids for the DA market

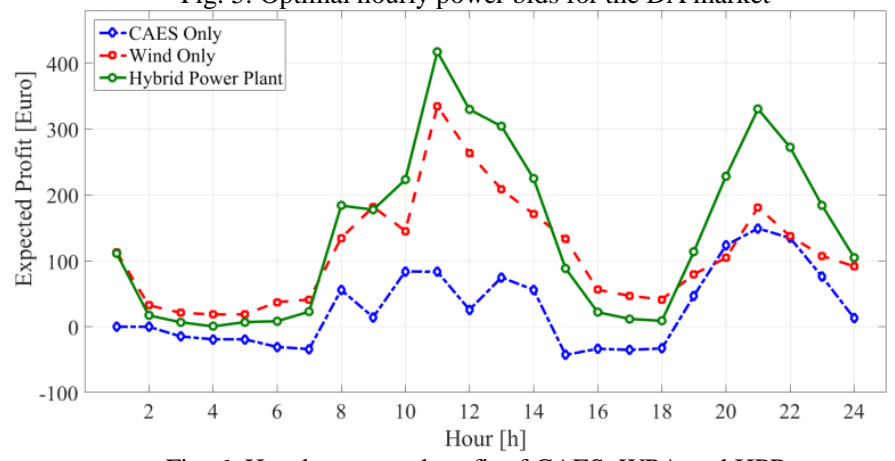

Fig. 6. Hourly expected profit of CAES, WPA and HPP

that more capacity of CAES for storage is exploited during the period of off-peak when it joins with WPA, and subsequently this stored energy can be released during the periods of offpeak. Obviously, more flexibility of production can be offered by having a HPP comparing to two independent aggregators to provide energy during specific peak periods. Hourly expected profit of operation for three given configurations are compared in Fig. 6. Noteworthy, WPA can offer higher hourly profit than HPP is some periods (e.g., hours 2 -7). It can be deduced that the CAES initially attempts to entirely exploit the wind power to store energy in its cavern, and then charges the remaining capacity by buying energy from the markets. On the contrary, hourly profit of HPP is superior to WPA in some periods (e.g. hours $19-24$ ) as the result of simultaneous utilization of WPA and CAES.

\section{B. Case II: Considering Bidding Curve}

CAES aggregator and the WPA are incorporated in this case study to participate in different markets regarding bidding curves. None of the financial risk factor and simple-cycle mode of CAES are considered in this section. It means that value zero is considered for the risk factor $\zeta$; while the formulations related to CAES simple-cycle mode are not modelled in market formulation. However, bidding curves equations (14), (26) and (37) are incorporated in the optimization. It can be inferred that by respect to the formulations of the bidding strategy, the opportunity for adoption of different patterns is available for CAES to tackle scenarios which model vacillations of DA market price.

As illustrations, bidding curves corresponding to some hours (i.e., 4, 7, 10, 14, 15, 17 and 19) of DA market are shown in Fig. 7. It is noticeable in the power curve corresponding to hour 10 that HPP bids zero for the prices less

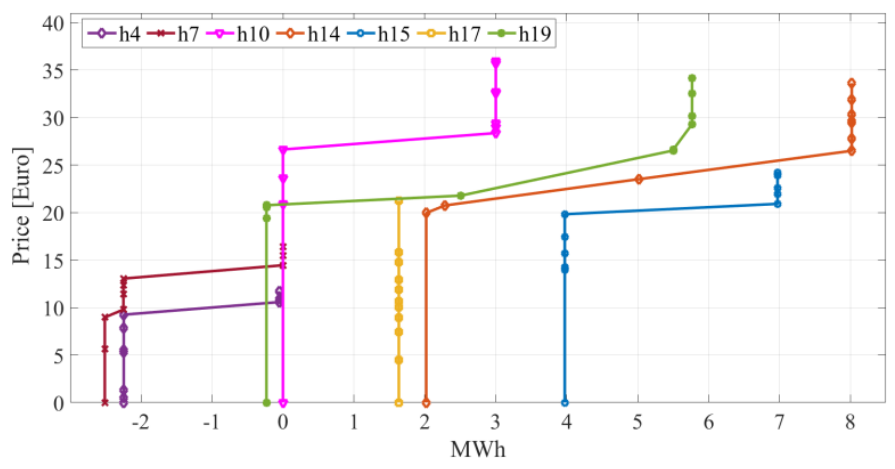

Fig. 7. HPP bidding curves for DA market

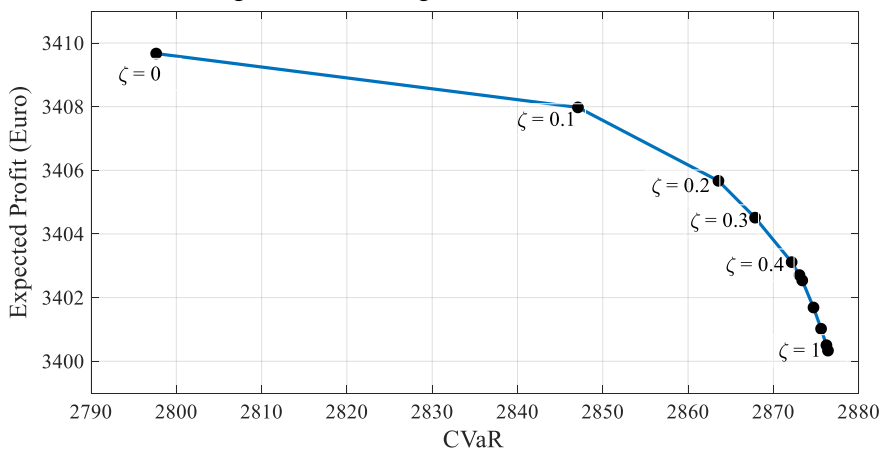

Fig. 8. Expected profit versus $\mathrm{CVaR}$ for different values of $\zeta$ : efficient frontier

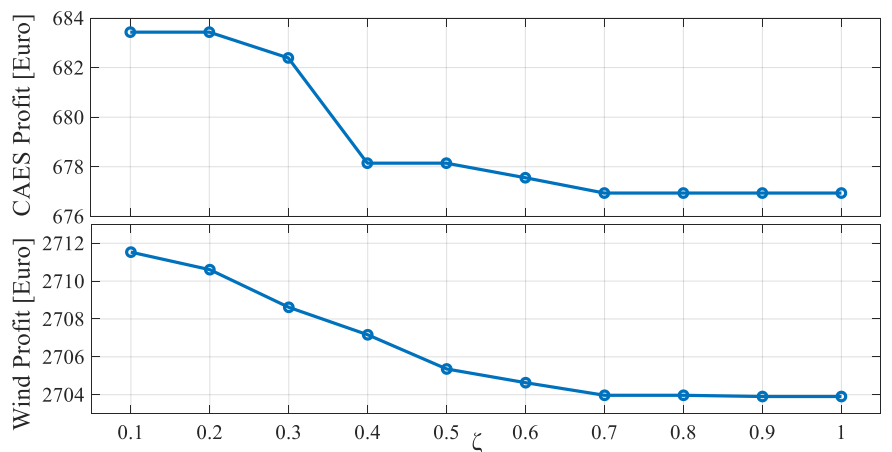

Fig. 9. Profit comparison for CAES and WPA versus different $\zeta$ values

than $€ 27$; while less than zero are bided for all prices in power curve related to hour 4 which means HPP tends to only buy power from the DA market. In fact, same pattern can be seen for hours 4 and 7 though zero-power is offered for the prices more than $€ 15$. Two pattern types can be seen in the bidding curve related to hour 19 . For this specific hour, there are 
buying of the power in terms of prices less than $€ 21$ and selling for prices more than $€ 21$. It should be mentioned that HPP tends to sell energy to the market for curves corresponding to hours 14,15 and 17. Straight line for offering curve of hour 17 means that the same amount of power is offered to the market by the HPP for all prices.

\section{Case III: Considering Financial Risk}

The coordination of CAES aggregator and WPA for participation in three mentioned markets is considered in this section while financial risk and bidding curves are considered. Modeling of simple-cycle mode of CAES is not included in this case. Equation (14), (26) and (37) are involved in the formulation of markets as similar to Case II.

Fig. 8 depicts the efficient frontier that is the expected profit contrasted with the $\mathrm{CVaR}$ for different values of $\zeta$. Obviously, the optimal solution achieved for $\zeta=0$ reaches the maximum expected profit and the maximum risk. The expected profit is different from $€ 3409.7$ for $\zeta=0$ to $€ 3400.3$ for $\zeta=1$, respectively. As it can be seen from Fig. 8, a decrease of $0.2738 \%$ in the expected profit produces $2.8153 \%$ increase in the CVaR. It is worthy to mention that moderately slight amount of the expected profit deviation and large amount of the CVaR specify a risk-averse solution. Also, low-risk solutions are the solutions with high CVaR and low expected profits. The patterns of profit change for configurations of WPA only and CAES only versus variations of $\zeta$ are shown in Fig. 9. Expected profit of HPP as well as its difference with profit summation of independent WPA and CAES operations as a function of $\zeta$ changes are shown in Fig. 10. As it can be deduced from Fig. 9 and Fig. 10, there are profit reductions for all configurations with the increase in value of risk factor $\zeta$. Obviously, such the decreases can be manifested as a reasonable and expectable phenomenon as the result of a decrease in amount of financial risk. However, HPP extra profit increases along with the growth of $\zeta$ value. In other words, more profit is attained even

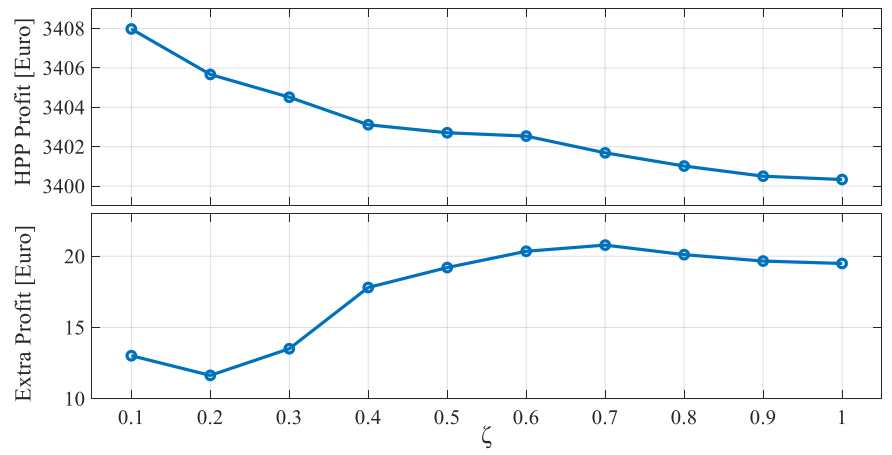

Fig. 10. HPP profit and its extra profit comparing to the summation of independent operations versus different $\zeta$ values

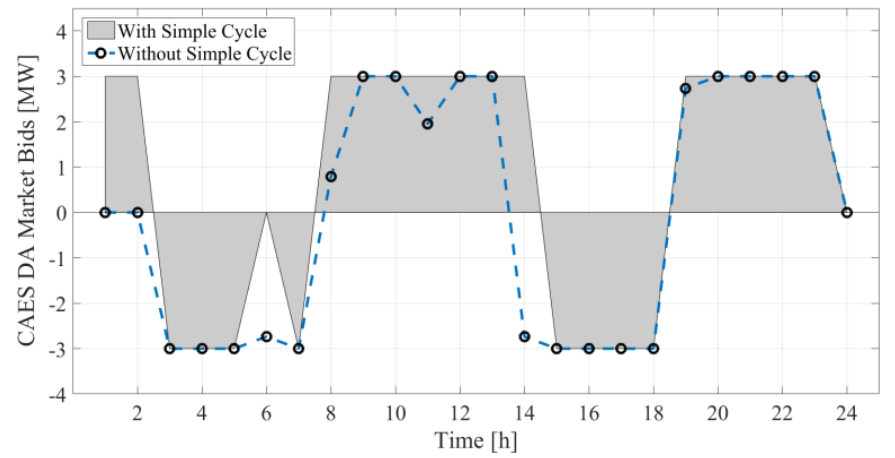

Fig. 11. Hourly power bids of CAES with/without CAES simple-cycle mode

when financial risk is considered in the joint operating model. Noteworthy that minimum and maximum extra profits are achieved at the risk factors $\zeta=0.2$ and $\zeta=0.7$ (i.e., equal to $€ 20.8317)$. In fact, it demonstrates the robustness of proposed joint configuration regarding the increase of financial risk of system.

\section{Case IV: Considering CAES Simple-cycle Mode}

This section investigates the coordination of CAES aggregator and WPA for participating in markets while considering the CAES simple-cycle mode, financial risk, and bidding curves. As similar to Case III, equations (14), (26) and (37) are incorporated in the problem formulation of markets. Besides, value 0.6 is assigned to the risk factor $\zeta$. Simplecycle mode of CAES is also added to the formulation.

Fig. 11 compares hourly power bids of CAES (with/without simple-cycle mode) to the DA market when it works as a producer in joint operation. It can be seen that CAES buys power from the market for fewer hours in the case of with the simple-cycle mode. Obviously, there is no need for CAES to charge the reservoir as much as when no simple-cycle mode is considered. Furthermore, the CAES can offer power to the market for two first hours of scheduling by utilizing the simple-cycle mode even though the cavern is in its initial minimum level (i.e. the cavern is empty at the starting point of scheduling). Simple-cycle mode is also can assist the system operator to fully exploit price strikes in the electricity market. In fact, HPP has the opportunity to employ the CAES simplecycle mode for an immediate power provision to the energy markets to attain the maximum profit of energy price fluctuations. As an illustration, this option is available when the power price is high for a specific period and no power can

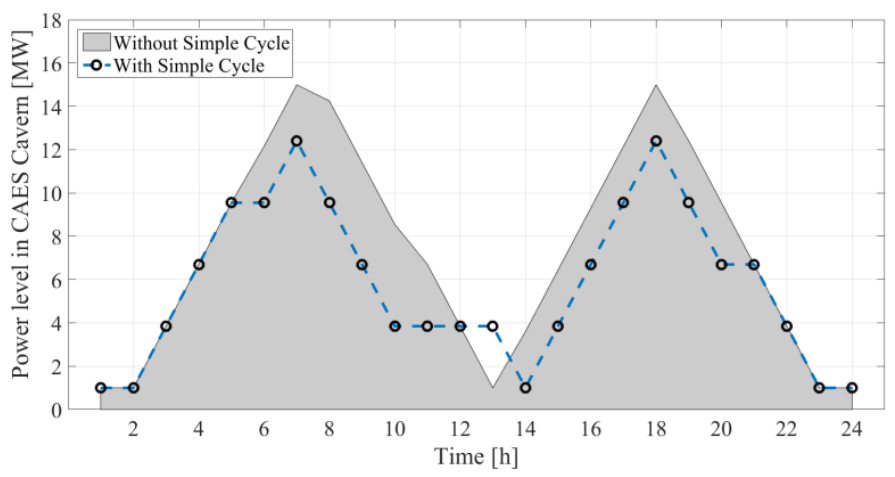

Fig. 12. Comparison of power level changes in the CAES cavern with/without CAES simple-cycle mode. 


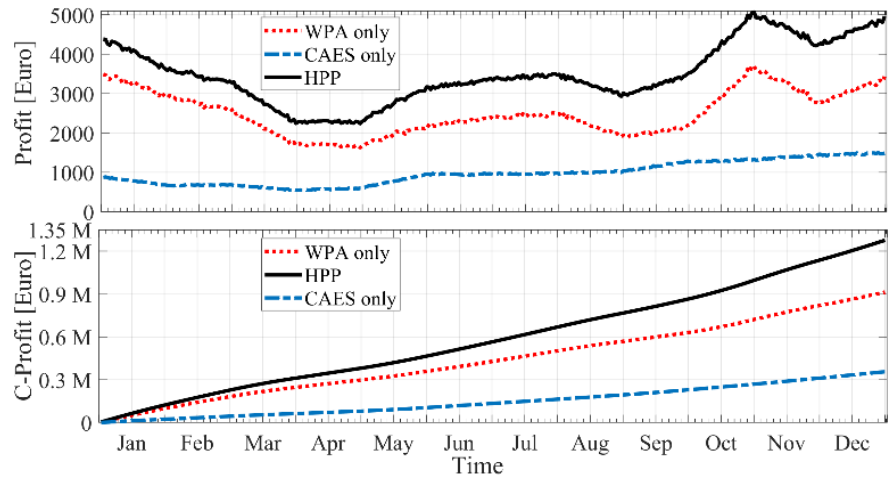

Fig. 13. Profit and cumulative profit (C-Profit) comparison for CAES only, WPA only and HPP over the course of one year

be provided by WPA (e.g., lack of wind) and CAES (e.g., there is no stored energy in cavern).

Fig. 12 compares changes of power level in the cavern of CAES when it operates with/without the simple-cycle mode in the joint configuration. It is shown that power level of the cavern does not reach its maximum when using simple-cycle mode, which means it is financially more beneficial to sell power in this mode (e.g., hour 6). In addition, some shifts can be observed in cavern depletion for some hours (e.g., 13 and 14) by means of simple-cycle mode that indicates more flexibility of the proposed approach.

\section{E. Case V: Case study over a one year period}

This section investigates the HPP for participating in markets while considering the CAES simple-cycle mode, financial risk, and bidding curves over the course of one year. Scenarios for the wind power and electricity prices are generated based on the historical data of wind speed and market prices in the Iberian Peninsula electricity market for the year 2016. Fig. 13 show the profit and cumulative profit (C-Profit) comparison for CAES only, WPA only and HPP over the course of one year. As it can be seen in Fig. 13, the daily profit of HPP is higher than the individual operation of WPA and CAES aggregators. Moreover, the cumulative profit of joint configuration is superior to the ones with independent configurations.

\section{CONCLUSION}

This paper has been proposed a methodology for joint operation of a CAES aggregator and a WPA which participates in DA, intraday and balancing markets in the form of a three-stage problem. This stochastic mixed-integer linear programming has been solved with an available commercial solver of GAMS software.

To adjust the operation of the HPP to the mentioned markets, both bidding curves and bidding quantity have been offered. Also, a simple-cycle mode has been added into the CAES operation to reach a more flexible operation for presented joint configuration in case of electricity price strike. Also, fewer hours of buying power from the market have been reached using CAES simple-cycle mode. Besides, CAES does not need to charge the cavern as much as when there is no simple-cycle mode. By using the join operation, the HPP has

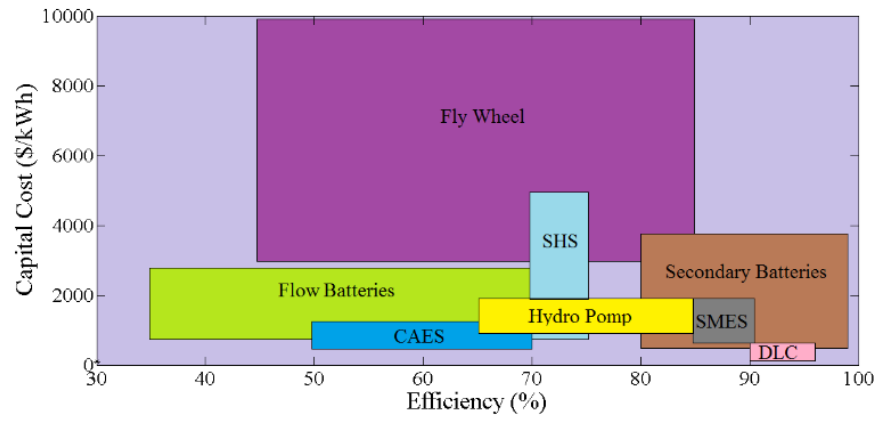

Fig. 14. Comparison of the efficiency and investment cost of different type of energy storage systems

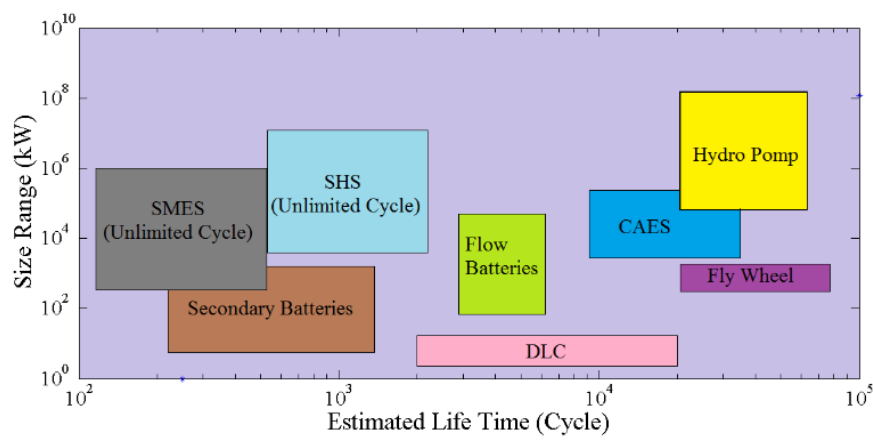

Fig. 15. Comparison of the life time and size range of different types of energy storages

achieved more profit even by considering the financial risk.

\section{APPENDIX}

The efficiency and investment cost of different type of energy storage systems are illustrated in Fig. 14. According to this figure, the CAES investment cost is less than the other large-scale types of energy storage systems. The efficiency of large-scale CAES is close to $70 \%$ and owing to the low investment cost, this efficiency level is fair. In addition, life time is one of important parameters of energy storages. Fig. 15 depicts the life time and size range of different types of energy storages. According to this figure, the CAES systems have a long life time duration in comparison with many other types of energy storage which are capable to be employed in power systems to integrate with renewable energy resources.

\section{REFERENCES}

[1] E. Fertig and J. Apt, "Economics of compressed air energy storage to integrate wind power: A case study in ERCOT," Energy Policy, vol. 39, no. 5, pp. 2330-2342, 2011.

[2] S. Shafiee, H. Zareipour, A. M. Knight, N. Amjady, and B. Mohammadi-Ivatloo, "Risk-Constrained Bidding and Offering Strategy for a Merchant Compressed Air Energy Storage Plant," IEEE Transactions on Power Systems, 2016.

[3] S. Shafiee, H. Zareipour, A. M. Knight, N. Amjady, and B. Mohammadi-Ivatloo, "Risk-constrained bidding and offering strategy for a merchant compressed air energy storage plant," IEEE Transactions on Power Systems, vol. 32, no. 2, pp. 946-957, 2017.

[4] M. Black and G. Strbac, "Value of bulk energy storage for managing wind power fluctuations," IEEE transactions on energy conversion, vol. 22, no. 1, pp. 197-205, 2007.

[5] L. Baringo and A. J. Conejo, "Offering strategy of wind-power producer: A multi-stage risk-constrained approach," IEEE Transactions on Power Systems, vol. 31, no. 2, pp. 1420-1429, 2016. 
[6] J. M. Morales, A. J. Conejo, and J. Pérez-Ruiz, "Short-term trading for a wind power producer," IEEE Transactions on Power Systems, vol. 25, no. 1, pp. 554-564, 2010.

[7] L. Baringo and A. J. Conejo, "Strategic offering for a wind power producer," IEEE Transactions on Power Systems, vol. 28, no. 4, pp. 4645-4654, 2013.

[8] M. Zugno, J. M. Morales, P. Pinson, and H. Madsen, "Pool strategy of a price-maker wind power producer," IEEE Transactions on Power Systems, vol. 28, no. 3, pp. 3440-3450, 2013.

[9] A. Rabiee, A. Soroudi, B. Mohammadi-Ivatloo, and M. Parniani, "Corrective voltage control scheme considering demand response and stochastic wind power," IEEE Transactions on Power Systems, vol. 29, no. 6, pp. 2965-2973, 2014.

[10] M. Güçyetmez and E. Çam, "A new hybrid algorithm with geneticteaching learning optimization (G-TLBO) technique for optimizing of power flow in wind-thermal power systems," Electrical Engineering, vol. 98, no. 2, pp. 145-157, 2016.

[11] M. S. Al-Swaiti, A. T. Al-Awami, and M. W. Khalid, "Co-optimized Trading of Wind-Thermal-Pumped Storage System in Energy and Regulation Markets," Energy, 2017.

[12] M. Asensio and J. Contreras, "Risk-constrained optimal bidding strategy for pairing of wind and demand response resources," IEEE Transactions on Smart Grid, vol. 8, no. 1, pp. 200-208, 2017.

[13] J. Garcia-Gonzalez, R. M. R. de la Muela, L. M. Santos, and A. M. Gonzalez, "Stochastic joint optimization of wind generation and pumped-storage units in an electricity market," IEEE Transactions on Power Systems, vol. 23, no. 2, pp. 460-468, 2008.

[14] A. A. S. de la Nieta, J. Contreras, and J. I. Munoz, "Optimal coordinated wind-hydro bidding strategies in day-ahead markets," IEEE Transactions on Power Systems, vol. 28, no. 2, pp. 798-809, 2013.

[15] J. B. Greenblatt, S. Succar, D. C. Denkenberger, R. H. Williams, and R. H. Socolow, "Baseload wind energy: modeling the competition between gas turbines and compressed air energy storage for supplemental generation," Energy Policy, vol. 35, no. 3, pp. 14741492, 2007.

[16] J. Mohammadi, A. Rahimi-Kian, and M.-S. Ghazizadeh, "Aggregated wind power and flexible load offering strategy," IET renewable power generation, vol. 5, no. 6, pp. 439-447, 2011.

[17] X. Zhang, "Optimal scheduling of critical peak pricing considering wind commitment," IEEE Transactions on Sustainable Energy, vol. 5, no. 2, pp. 637-645, 2014.

[18] E. Heydarian-Forushani, M. P. Moghaddam, M. K. Sheikh-El-Eslami, M. Shafie-khah, and J. P. Catalão, "Risk-constrained offering strategy of wind power producers considering intraday demand response exchange," IEEE Transactions on sustainable energy, vol. 5, no. 4, pp. 1036-1047, 2014

[19] S. Ghavidel et al., "Hybrid power plant bidding strategy including a commercial compressed air energy storage aggregator and a wind power producer," in Universities Power Engineering Conference (AUPEC), 2017 Australasian, 2017, pp. 1-6: IEEE.

[20] A. J. Conejo, M. Carrión, and J. M. Morales, Decision making under uncertainty in electricity markets. Springer, 2010.

[21] J. F. Dean, "The Art \& Science of Financial Risk Analysis," 2006. NACM

[22] J. Aghaei, M. Barani, M. Shafie-Khah, A. A. S. de la Nieta, and J. P. Catalão, "Risk-constrained offering strategy for aggregated hybrid power plant including wind power producer and demand response provider," IEEE Transactions on Sustainable Energy, vol. 7, no. 2, pp. 513-525, 2016.

[23] S. Ghavidel, A. Azizivahed, and L. Li, "A hybrid Jaya algorithm for reliability-redundancy allocation problems," Engineering Optimization, vol. 50, no. 4, pp. 698-715, 2018.

[24] "Sotavento Wind Farm [Online]. Available: http://www.sotaventogalicia.com/."

[25] N. Amjady, J. Aghaei, and H. A. Shayanfar, "Stochastic multiobjective market clearing of joint energy and reserves auctions ensuring power system security," IEEE Transactions on Power Systems, vol. 24, no. 4, pp. 1841-1854, 2009.

[26] "Red Eléctrica de España, e-sios [Online]. Available: http://www.esios.ree.es/web-publica."

[27] A. Ben-Tal, L. El Ghaoui, and A. Nemirovski, Robust optimization. Princeton University Press, 2009.

[28] Y. Ben-Haim, Info-gap decision theory: decisions under severe uncertainty. Elsevier, 2006. 